\title{
Obesity cutoff values in Korean men with motor complete spinal cord injury: body mass index and waist circumference
}

\author{
Jae-Hyun Yun ${ }^{1}$ Seong-Min Chun ${ }^{2} \cdot$ Ju-Chan Kim ${ }^{1} \cdot$ Hyung-lk Shin ${ }^{1}$
}

Received: 1 March 2018 / Revised: 23 May 2018 / Accepted: 12 June 2018 / Published online: 5 July 2018

(c) International Spinal Cord Society 2018

\begin{abstract}
Study design Cross-sectional.

Objectives To determine the obesity cutoff values for body mass index (BMI) and waist circumference (WC) in Korean men with motor complete spinal cord injury (SCI).

Setting Tertiary level hospital in Seoul, Korea.

Methods BMI $\left(\mathrm{kg} / \mathrm{m}^{2}\right)$ was calculated and WC $(\mathrm{cm})$ was measured in each participant in the supine position. Percentage of total body fat (\%) was determined using whole-body dual-energy X-ray absorptiometry. Receiver operating characteristic curves were generated to determine the obesity cutoff values for BMI and WC. The optimal cutoff values were determined using the Youden index. Identified cutoff values were compared with those of 195 age- and BMI-matched men in the general population (GP), obtained from the Korea National Health and Nutrition Examination Survey.

Results This study included 52 Korean men with motor complete SCI. A BMI of $20.2 \mathrm{~kg} / \mathrm{m}^{2}$ and WC of $81.3 \mathrm{~cm}$ were identified as obesity cutoff values in Korean men with motor complete SCI. The area under the curve (AUC) was 0.928, specificity was $100 \%$, and sensitivity was $84.8 \%$ according to the ROC curve of BMI for participants. AUC was 0.964 , specificity was $100 \%$, and sensitivity was $84.8 \%$ in the ROC curve of WC for participants. A BMI of $22.5 \mathrm{~kg} / \mathrm{m}^{2}$ and WC of $85.5 \mathrm{~cm}$ were identified as obesity cutoff values in age- and BMI-matched men in the GP.

Conclusions The obesity cutoff values in Korean men with motor complete SCI that were determined in this study were lower than those of the Korean GP and western people with SCI.
\end{abstract}

\section{Introduction}

Obesity is the clinical manifestation of excess of fat tissue and releases a lot of biochemical substances such as leptin. Increased fat tissue affects the insulin resistance, inflammatory condition, and hormonal homeostasis and results in obesity-associated complications like cardiovascular disease (CVD) [1]. Therefore, it is important to measure body fat mass to assess obesity.

To evaluate the body composition and body fat mass, several methods like underwater immersion and body

Hyung-Ik Shin

hyungik1@snu.ac.kr

1 Department of Rehabilitation Medicine, Seoul National University Hospital, Seoul, Korea

2 Department of Rehabilitation Medicine, Purme Children's Rehabilitation Hospital, Seoul, Korea plethysmography are needed but are difficult to apply in clinical practice. Although dual-energy X-ray absorptiometry (DXA), magnetic resonance imaging (MRI), and computerized tomography (CT) are thought to be clinically more applicable, these are being used for research purposes rather than clinical assessment [2]. For this reason, the surrogate measures that can be used easily to assess fat mass are needed. Among the surrogate measures, waist circumference (WC) and body mass index (BMI) are generally used. Therefore, it is important to establish the cutoff value of WC and BMI for excessive body fat mass in determining whether to start management for obesity in clinical environment.

The cutoff values for obesity in WC and BMI are different for sex, race, and specific population. Especially, several previous studies have demonstrated that the body composition of people with spinal cord injury (SCI) differs from that of the general population (GP), and the percentage of total body fat (PBF) was reported to be $8-18 \%$ higher in people with SCI than in their age-, height-, and weight- 
matched subjects from the GP [3-6]. Therefore, obesity can be underestimated in people with SCI when the cutoff value of obesity in the GP is applied.

Because of these concerns, efforts have been made to establish cutoff values in people with SCI for accurate assessment of obesity in western countries. Laughton et al. [7] recommended that the BMI cutoff value for obesity in western people with SCI should decrease to $22.0 \mathrm{~kg} / \mathrm{m}^{2}$, which is lower than the cutoff value recommended by the World Health Organization (WHO) for non-Asian GP, $30.0 \mathrm{~kg} / \mathrm{m}^{2}$. In addition, some epidemiologic studies of normal Asian populations have shown that a high PBF with a low BMI and WC is more likely to occur in Asians than in the western population [8-11]. Therefore, CVD risk factors and comorbidities could be more probable in the Asian population than in other ethnic groups, even with a low BMI or WC $[8,12]$. For this reason, applying cutoff values for western people with SCI to the Asian population is not appropriate, and it is important to establish Asian-specific cutoff values for obesity for people with SCI. However, reports on obesity cutoff values in Asian individuals with SCI remain scarce [2]. The present study aimed to determine the obesity cutoff values for BMI and WC in Korean men with motor complete SCI based on body composition analysis.

\section{Methods}

\section{Study population}

This study included people with SCI who visited a tertiary level general hospital outpatient clinic between November 2013 and April 2015. The inclusion criteria of this study were as follows: (1) men with motor complete SCI, (2) age over 18 years, and (3) injury duration over 12 months. Individuals with other systemic diseases that could cause weight loss or muscle deterioration, such as diabetes, cancer, and/or any other chronic illnesses, were excluded. Demographic data, including age, location and level of the lesion, and injury duration (in years), were recorded for each participants by trained interviewers using a standardized assessment form (American Spinal Injury Association Impairment Scale, AIS). An experienced rehabilitation physician classified each injury according to the level and severity.

The data for 195 age- and BMI-matched $( \pm 3$ years, \pm $1 \mathrm{~kg} / \mathrm{m}^{2}$ ) men in the GP were obtained from the Korea National Health and Nutrition Examination Survey (KNHANES) 2011, by using the greedy method.

\section{BMI}

Weight $(\mathrm{kg})$ and height $(\mathrm{m})$ were measured in each participant. Weight was measured using a digital wheelchair scale (Kyongin Medical, Seoul, Korea), and the weight of the wheelchair was subtracted from the total weight of the participant and wheelchair. Height was measured in the supine position by the physiatrist. The heights of participants with ankle plantar flexion contracture were measured from the heel to the top of the head. Since participants with knee flexion contracture could not keep their legs straight, the leg sections were divided into the following two sections: (1) from the hip joint to knee joint and (2) from the knee joint to ankle joint. The length of each section was measured and summed. BMI was calculated by dividing weight by height squared $\left(\mathrm{m}^{2}\right)$.

\section{WC}

WC was measured at the end of normal expiration with the participants in the supine position using a flexible measuring tape that was placed at the level midway between the lowest rib and iliac crest; the recorded value was rounded to the nearest $0.1 \mathrm{~cm}[13,14]$. WC was measured by an experienced physician twice, and the mean value was used for analysis. The variability between the measurements was $<0.1 \mathrm{~cm}$ in all participants

In the KNHANES, WC was measured in the same method for all participants, except that they were measured in upright position with the arms hanging freely.

\section{Body fat mass}

Total body fat mass $(\mathrm{kg})$ and PBF were determined using DXA (Lunar Prodigy; GE Healthcare, Madison, WI, USA). Participants were transferred from the wheelchair to an adjustable height stretcher car with the help of two persons and then moved to the scanner bed using a transfer board. A trained technician performed all scans using the Lunar software (version 13.31) to measure PBF. Obesity was defined as a PBF of $23 \%$ or greater for those aged 20-39 years and 24\% or greater for those aged over 40 years [10].

In the KNHANES, PBFs were measured on different DXA instruments (Discovery QDR-4500W; Hologic Inc. Bedford, Massachusetts, USA) and APEX software. A number of studies reported that there is a significant difference between the PBF values measured by the two devices, therefore KNHANES data were converted to Lunar instrument using the published equation: $\mathrm{PBF}_{\mathrm{Lunar}}=$ $-2.173+1.119 \mathrm{PBF}_{\text {Hologic }}[15-18]$.

\section{Statistical analyses}

Data are presented with mean, standard deviation, 95\% confidence interval (CI), and median. The Shapiro-Wilk test was used to assess the normality of the data. Pearson's 


\begin{tabular}{|c|c|c|c|c|c|}
\hline & \multicolumn{2}{|l|}{$\mathrm{SCI}(N=52)$} & \multicolumn{2}{|l|}{$\mathrm{GP}(N=195)$} & \multirow[t]{2}{*}{$P$-value } \\
\hline & Mean \pm SD $(95 \%$ CI $)$ & Median (IQR) & Mean \pm SD $(95 \% \mathrm{CI})$ & Median (IQR) & \\
\hline Age (years) & $\begin{array}{l}42.1 \pm 11.4 \\
(38.9-45.3)\end{array}$ & $\begin{array}{l}39.0 \\
(32.0-51.0)\end{array}$ & $\begin{array}{l}42.8 \pm 11.3 \\
(41.2-44.4)\end{array}$ & $\begin{array}{l}40.0 \\
(33.0-52.0)\end{array}$ & 0.710 \\
\hline BMI $\left(\mathrm{kg} / \mathrm{m}^{2}\right)$ & $\begin{array}{l}22.6 \pm 2.9 \\
(21.8-23.4)\end{array}$ & $\begin{array}{l}22.1 \\
(20.2-25.1)\end{array}$ & $\begin{array}{l}22.9 \pm 2.7 \\
(22.5-23.3)\end{array}$ & $\begin{array}{l}22.0 \\
(21.0-25.0)\end{array}$ & 0.233 \\
\hline $\mathrm{WC}(\mathrm{cm})$ & $\begin{array}{l}88.1 \pm 9.9 \\
(85.3-90.9)\end{array}$ & $\begin{array}{l}88.4 \\
(81.2-97.1)\end{array}$ & $\begin{array}{l}80.9 \pm 10.2 \\
(79.5-82.3)\end{array}$ & $\begin{array}{l}80.0 \\
(75.0-88.0)\end{array}$ & $<750.01$ \\
\hline FM (kg) & $\begin{array}{l}22.9 \pm 7.2 \\
(20.9-24.9)\end{array}$ & $\begin{array}{l}23.3 \\
(17.7-28.6)\end{array}$ & $\begin{array}{l}13.9 \pm 5.1 \\
(13.2-14.6)\end{array}$ & $\begin{array}{l}13.3 \\
(10.2-17.0)\end{array}$ & $<100.01$ \\
\hline PBF (\%) & $\begin{array}{l}32.8 \pm 7.7 \\
(30.6-34.9)\end{array}$ & $\begin{array}{l}33.5 \\
(28.2-38.3)\end{array}$ & $\begin{array}{l}20.4 \pm 5.5 \\
(19.6-21.2)\end{array}$ & $\begin{array}{l}20.0 \\
(16.0-24.0)\end{array}$ & $<160.01$ \\
\hline
\end{tabular}

$B M I$ body mass index, $C I$ confidence interval, $F M$ fat mass, $G P$ age- and BMI- matched men in general population, $I Q R$ interquartile range, $P B F$ percentage of total body fat, $S C I$ Patient with spinal cord injury, $S D$ standard deviation, $W C$ waist circumference

product-moment analyses were performed to determine the correlations of BMI and WC with PBF.

Receiver operating characteristic (ROC) curves were generated to determine the obesity cutoff values of BMI and WC for people with SCI and age- and BMI-matched men in the GP. The optimal cutoff values were determined using the Youden index: maximum $=$ insensitivity + enspecificity -1 . This index has a range from 0 to 1 , with the values closer to 1 indicating that the biomarker's effectiveness is relatively large, while the values closer to 0 indicate limited effectiveness. The Youden index uses the maximum vertical distance of the ROC curve from a specific point on the diagonal line (i.e., the chance line) [19].

All statistical analyses were performed using Statistical Package for the Social Sciences version 23.0 (IBM Corp., Armonk, NY, USA). A $P$-value $<0.05$ was considered to be statistically significant.

\section{Results}

\section{Participants characteristics}

The characteristics of the participants are presented in Table 1. The study included 52 Korean men with motor complete SCI. The mean (SD) age of the participants was 42.1 (11.4) years, and the mean (SD) injury duration was 13.2 (8.0) years. Of the 52 individuals with SCI, 35 had a cervical lesion, while 17 had a thoracic lesion. All participants had motor complete SCI according to the AIS, with 33 individuals classified as $\mathrm{A}$ and 19 classified as B.

\section{BMI}

The mean (SD) BMI was $22.6(2.9) \mathrm{kg} / \mathrm{m}^{2}$ for men with SCI and $22.9(2.7) \mathrm{kg} / \mathrm{m}^{2}$ for age- and BMI-matched able-bodied men. Both data were normally distributed. BMI was significantly correlated with $\mathrm{PBF}(r=0.509, P<0.0001$, for men with SCI; $r=0.577, P<0.0001$, for age- and BMImatched able-bodied men) (Fig. 1a, b). According to the ROC curve of BMI for men with SCI, the area under the curve (AUC) was 0.928, and the optimal cutoff value was $20.2 \mathrm{~kg} / \mathrm{m}^{2}$. Specificity was $100 \%$, and sensitivity was $84.8 \%$ (95\% CI, 0.851-1.000; $P<0.0001)$. In contrast, according to the ROC curve of age- and BMI-matched men in the GP, AUC was 0.789, and the cutoff value was calculated as $22.5 \mathrm{~kg} / \mathrm{m}^{2}$. Specificity was $66.7 \%$, and sensitivity was $81.7 \%(95 \% \mathrm{CI}, \quad 0.741-0.861 ; P<0.0001)$ (Fig. 1c, d). When the BMI cutoff value of $20.2 \mathrm{~kg} / \mathrm{m}^{2}$ was applied for obesity, $75 \%$ (39/52) of participants were identified as being obese (95\% CI, 63-87\%). However, when the cutoff value of matched men in the GP $(22.5 \mathrm{~kg} /$ $\mathrm{m}^{2}$ ) was applied, $44 \%(23 / 52)$ of participants were identified as being obese (95\% CI, 31-57\%).

\section{WC}

The mean (SD) WC was 88.1 (9.9) $\mathrm{cm}$ for men with SCI and 80.9 (10.2) cm for age- and BMI-matched able-bodied men. Both data were normally distributed. WC was also correlated with PBF PBF $(r=0.711, P<0.0001$, for men with SCI; $r=0.514, P<0.0001$, for age-and BMI-matched able-bodied men) (Fig. 2a, b). In the ROC curve of WC for men with SCI, the AUC was 0.964, and the optimal cutoff value was identified as $81.3 \mathrm{~cm}$. Specificity was $100 \%$, and sensitivity was $84.8 \%$ (95\% CI, 0.908-1.000; $P<0.0001)$. In the ROC curve of WC for matched able-bodied men, the AUC was 0.791 , and the cutoff value was calculated as $85.5 \mathrm{~cm}$. Specificity was $85.2 \%$, and sensitivity was $61.7 \%$ (95\% CI, 0.722-0.860; $P<0.0001$ ) (Fig. 2c, d). When applying the WC cutoff value of $81.3 \mathrm{~cm}, 75 \%(39 / 52)$ of participants were considered obese (95\% CI, 63-87\%). 

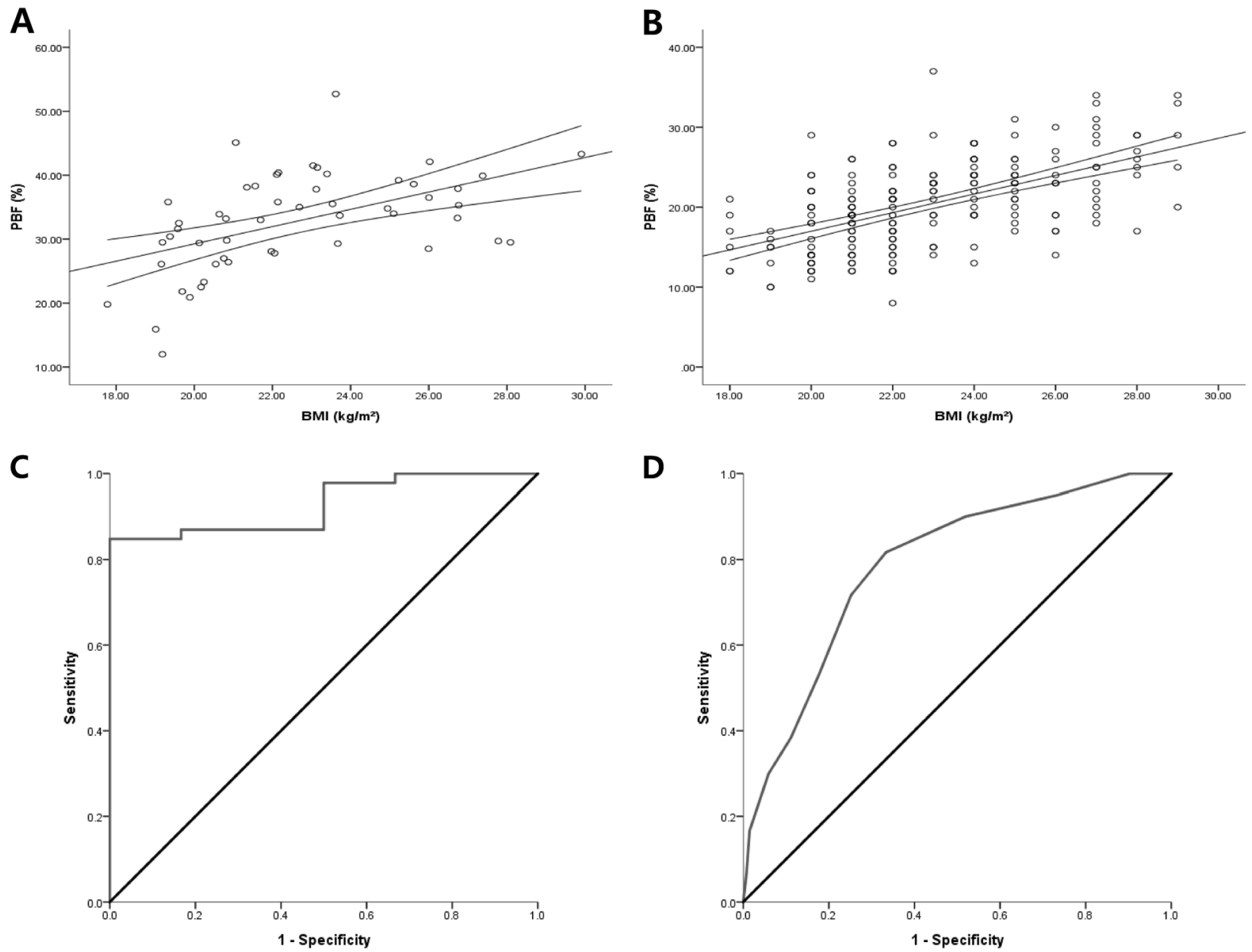

Fig. 1 Scatter plots and receiver operating characteristic (ROC) curve of body mass index (BMI) of 52 participants and age- and BMImatched 195 men in the general population (GP). Pearson's productmoment analyses were performed to determine the correlations of BMI with percentage of body fat $(\mathrm{PBF})$. ROC curves were generated to determine the obesity cutoff values of BMI. The area under the ROC

When applying the cutoff value of matched able-bodied men in the GP $(85.5 \mathrm{~cm}), 60 \%$ (31/52) of participants were considered obese $(95 \% \mathrm{CI}, 47-73 \%)$.

\section{Discussion}

We identified the cutoff value of BMI as $20.2 \mathrm{~kg} / \mathrm{m}^{2}$ and WC as $81.3 \mathrm{~cm}$ for obesity in Korean men with SCI. The calculated cutoff value for age- and BMI-matched ablebodied men was $22.5 \mathrm{~kg} / \mathrm{m}^{2}$ for BMI and $85.5 \mathrm{~cm}$ for WC. Identified cutoff values of BMI and WC were lower than those of matched men in the GP. Moreover, as shown in Table 1, PBF was different between the SCI group and matched men in the GP. These results were consistent with other studies that reported that people with SCI have higher $\mathrm{PBF}$ and lower cutoff values than the GP [3,5-7]. When cutoff values of age- and BMI-matched men in the GP were

curves are shown. The optimal cutoff values were determined using the Youden index. a The correlation between BMI and PBF of participants. $\mathbf{b}$ The correlation between BMI and PBF of age- and BMImatched men in the GP. Regression lines and confidence intervals are shown. $\mathbf{c}$ The ROC curve of BMI for participants. $\mathbf{d}$ The ROC curve of BMI for age- and BMI-matched men in the GP

applied to people with SCI, a number of obese participants could be considered non-obese. They might be neglected, therefore not receiving adequate management for obesity.

In addition, the identified cutoff value for BMI $\left(20.2 \mathrm{~kg} / \mathrm{m}^{2}\right)$ was lower than that for western people with SCI $\left(22.0 \mathrm{~kg} / \mathrm{m}^{2}\right)$, as reported by Laughton et al. [7], and the identified cutoff value for WC $(81.3 \mathrm{~cm})$ was lower than that for western people with SCI $(94 \mathrm{~cm})$, as suggested by Ravensbergen et al. [20]. This study showed that considerably lower anthropometric surrogate obesity cutoff values should be applied to Asian people with SCI compared with the GP and western people with SCI.

Inayama et al. [21] suggested a WC cutoff value of 81.3 $\mathrm{cm}$ for abdominal obesity in Japanese men with SCI through measuring visceral fat area using computed tomography (CT). The abovementioned WC cutoff value $(81.3$ $\mathrm{cm}$ ) is the same as that identified in the present study. At least in part, this similarity might be explained by a smaller 

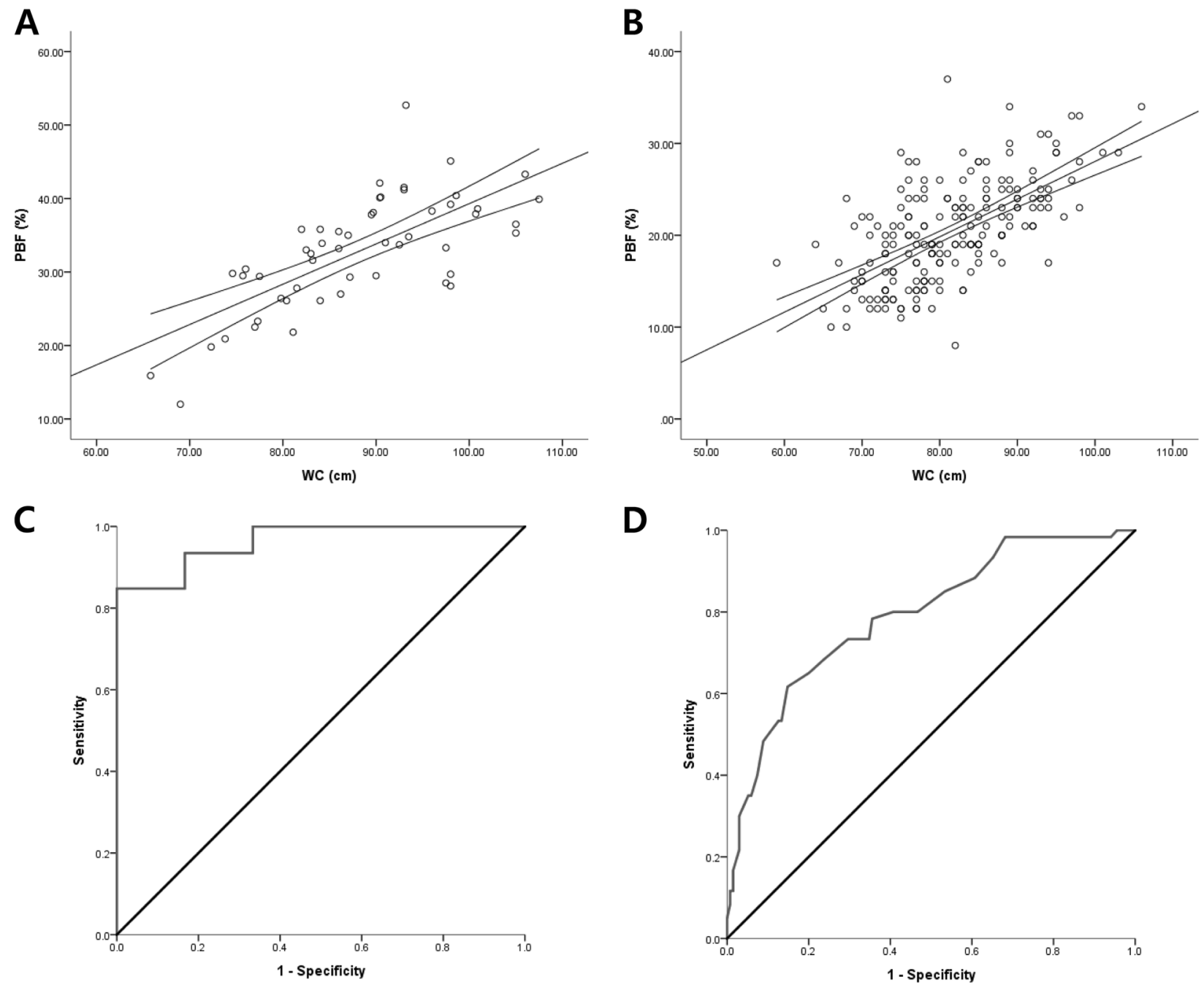

Fig. 2 Scatter plots and receiver operating characteristic (ROC) curve of waist circumference (WC) of 52 participants and age- and body mass index- matched 195 men in the general population (GP). Pearson's product-moment analyses were performed to determine the correlations of WC with percentage of body fat (PBF). ROC curves were generated to determine the obesity cutoff values of WC. The area

ethnic difference between Korean and Japanese populations compared to the likely differences between Korean and western populations.

The reason why the PBF of people with SCI tends to be higher than GP is thought to be that people with SCI experience changes in body composition, metabolic rate, and autonomic function and have a sedentary lifestyle after injury $[22,23]$. The reason for the difference in PBF from western and Asian people is not clear, but it could be explained by their physical differences. Asian people have relatively shorter legs and slender body shape so they are more likely to have less muscles and connective tissues but more body fat mass than western people [8]. Thus, Asian people have higher PBF with relatively lower BMI and WC. It could underestimate obesity, therefore increasing the

under the ROC curves are shown. The optimal cutoff values were determined using the Youden index. a The correlation between WC and PBF of participants. b The correlation between WC and PBF of age- and BMI- matched men in the GP. Regression lines and confidence intervals are shown. $\mathbf{c}$ The ROC curve of WC for participants. d The ROC curve of WC for age- and BMI- matched men in the GP

development of obesity-related complications like CVD $[8,12]$.

In age- and BMI-matched able-bodied men, WC was measured in the standing position after normal expiration with the arms hanging freely. However, all people with motor complete SCI were unable to independently maintain a standing posture; therefore, WC was measured while lying in the supine position in the present study and previous research. However, Eriks-Hoogland et al. [24] chose a $30^{\circ}$ supine position in order to mimic the standing position as much as possible. Furthermore, no standardized WC measurement protocols indicating the exact location of the measuring tape are available. Some researchers measured $\mathrm{WC}$ at the narrowest part of the waist after normal expiration $[20,24,25]$, while others measured $\mathrm{WC}$ at the level of 
the umbilicus $[21,26]$ or at the midpoint between the top of the iliac crest and the lower margin of the last palpable rib in the midaxillary line $[13,14]$. In the present study, we chose the midpoint between the top of the iliac crest and the lower margin of the last palpable rib for the following reasons. First, the umbilicus may not be an appropriate landmark in persons with obesity as this position changes with increases in fat mass [27]. Second, in many persons with SCI, the narrowest waist seemed to be at the lowest rib [27]. WCs of age- and BMI-matched men in the KNHANES data were measured in the same method except that they were measured in the standing position.

The present study had limitations. First, this is a sample of convenience, so it may not be truly representative of the target population. Especially, there was a very wide postinjury duration distribution (median 11.4 years, IQR = 7.0-20.4). Gorgey et al. [28] reported that there was no correlation between PBF and post-injury duration $(r=0.16$, $P=0.3$ ). Second, we only included participants with motor complete SCI; therefore, it could be inappropriate to use the obesity cutoff values identified in this study in people with incomplete injury. Third, due to the relatively small number of participants, we did not provide the cutoff values according to the lesion level (paraplegia vs. tetraplegia) and for women.

\section{Conclusion}

In conclusion, this study identified a BMI cutoff value of $20.2 \mathrm{~kg} / \mathrm{m}^{2}$ and a WC cutoff value of $81.3 \mathrm{~cm}$ for obesity in Korean men with motor complete SCI, which are much lower than those of western people with SCI and Korean men in the GP. The findings from this study can help evaluate obese people with motor complete SCI who might be regarded as non-obese when the cutoff values for the GP or western people with SCI were applied.

Acknowledgements We thank Hye-Ri Kim, Il-Young Jung, and Ja-Ho Leigh for contributing to the data collection. This study was supported by the Bio \& Medical Technology Development Program of the National Research Foundation of Korea.

Author contributions JHY was responsible for extracting and analyzing data, interpreting results, creating the figures and writing the report. He contributed to designing the study and collecting data. SMC was responsible for designing the study, writing the initial protocol, collecting data. He contributed to interpreting results and writing the report. JCK was responsible for analyzing data, writing the report and updating reference lists. He contributed to collecting data and interpreting the results. HIS was responsible for designing the study, collecting data, interpreting the results. He provided feedback on the report and approved the final version.

Funding This study was funded by the Ministry of Science, ICT, and Future Planning (2013R1A1A2010246).

\section{Compliance with ethical standards}

Statement of ethics All applicable institutional and governmental regulations concerning the ethical use of human volunteers were followed during the course of this research. The study was approved by the Institutional Review Board of Seoul National Hospital (IRB No. 1412-115-634), and written informed consent was obtained from all study participants.

Conflict of interest The authors declare that they have no conflict of interest.

\section{References}

1. Van Gaal LF, Mertens IL, De Block CE. Mechanisms linking obesity with cardiovascular disease. Nature. 2006;444:875-80.

2. Silveira SL, Ledoux TA, Robinson-Whelen S, Stough R, Nosek MA. Methods for classifying obesity in spinal cord injury: a review. Spinal Cord. 2017;55:812-7.

3. Monroe MB, Tataranni PA, Pratley R, Manore MM, Skinner JS, Ravussin E. Lower daily energy expenditure as measured by a respiratory chamber in subjects with spinal cord injury compared with control subjects. Am J Clin Nutr. 1998;68:1223-7.

4. Buchholz AC, McGillivray CF, Pencharz PB. The use of bioelectric impedance analysis to measure fluid compartments in subjects with chronic paraplegia. Arch Phys Med Rehabil. 2003;84:854-61.

5. Spungen AM, Adkins RH, Stewart CA, Wang J, Pierson RN Jr., Waters RL, et al. Factors influencing body composition in persons with spinal cord injury: a cross-sectional study. J Appl Physiol. 2003;95:2398-407.

6. Jones LM, Legge M, Goulding A. Healthy body mass index values often underestimate body fat in men with spinal cord injury. Arch Phys Med Rehabil. 2003;84:1068-71.

7. Laughton GE, Buchholz AC, Martin Ginis KA, Goy RE, Group SSR. Lowering body mass index cutoffs better identifies obese persons with spinal cord injury. Spinal Cord. 2009; 47:757-62.

8. Deurenberg P, Deurenberg-Yap M, Guricci S. Asians are different from Caucasians and from each other in their body mass index/ body fat per cent relationship. Obes Rev. 2002;3:141-6.

9. Wang J, Thornton JC, Russell M, Burastero S, Heymsfield S, Pierson RN Jr. Asians have lower body mass index (BMI) but higher percent body fat than do whites: comparisons of anthropometric measurements. Am J Clin Nutr. 1994;60:23-8.

10. Gallagher D, Heymsfield SB, Heo M, Jebb SA, Murgatroyd PR, Sakamoto Y. Healthy percentage body fat ranges: an approach for developing guidelines based on body mass index. Am J Clin Nutr. 2000;72:694-701.

11. Wang J, Thornton JC, Burastero S, Shen J, Tanenbaum S, Heymsfield SB, et al. Comparisons for body mass index and body fat percent among Puerto Ricans, blacks, whites and Asians living in the New York City area. Obes Res. 1996;4:377-84.

12. Consultation WHOE. Appropriate body-mass index for Asian populations and its implications for policy and intervention strategies. Lancet. 2004;363:157-63.

13. Cirnigliaro CM, LaFountaine MF, Dengel DR, Bosch TA, Emmons RR, Kirshblum SC, et al. Visceral adiposity in persons with chronic spinal cord injury determined by dual energy X-ray absorptiometry. Obesity. 2015;23:1811-7.

14. Nishida C, Ko GT, Kumanyika S. Body fat distribution and noncommunicable diseases in populations: overview of the 2008 WHO Expert Consultation on Waist Circumference and WaistHip Ratio. Eur J Clin Nutr. 2010;64:2-5. 
15. Shepherd JA, Fan B, Lu Y, Wu XP, Wacker WK, Ergun DL, et al. A multinational study to develop universal standardization of whole-body bone density and composition using GE Healthcare Lunar and Hologic DXA systems. J Bone Miner Res. 2012;27:2208-16.

16. Pearson D, Horton B, Green DJ. Cross calibration of Hologic QDR2000 and GE lunar prodigy for whole body bone mineral density and body composition measurements. J Clin Densitom. 2011;14:294-301.

17. Xu W, Chafi H, Guo B, Heymsfield SB, Murray KB, Zheng J, et al. Quantitative comparison of 2 dual-energy X-ray absorptiometry systems in assessing body composition and bone mineral measurements. J Clin Densitom. 2016;19:298-304.

18. Malouf J, DiGregorio S, Del Rio L, Torres F, Marin AM, Farrerons $\mathrm{J}$, et al. Fat tissue measurements by dual-energy $\mathrm{x}$-ray absorptiometry: cross-calibration of 3 different fan-beam instruments. J Clin Densitom. 2013;16:212-22.

19. Perkins NJ, Schisterman EF. The inconsistency of "optimal" cutpoints obtained using two criteria based on the receiver operating characteristic curve. Am J Epidemiol. 2006;163:670-5.

20. Ravensbergen HR, Lear SA, Claydon VE. Waist circumference is the best index for obesity-related cardiovascular disease risk in individuals with spinal cord injury. J Neurotrauma. 2014;31: 292-300.

21. Inayama $\mathrm{T}$, Higuchi $\mathrm{Y}$, Tsunoda $\mathrm{N}$, Uchiyama $\mathrm{H}$, Sakuma $\mathrm{H}$. Associations between abdominal visceral fat and surrogate measures of obesity in Japanese men with spinal cord injury. Spinal Cord. 2014;52:836-41.
22. Rajan S, McNeely MJ, Warms C, Goldstein B. Clinical assessment and management of obesity in individuals with spinal cord injury: a review. J Spinal Cord Med. 2008;31:361-72.

23. Gorgey AS, Dolbow DR, Dolbow JD, Khalil RK, Castillo C, Gater DR. Effects of spinal cord injury on body composition and metabolic profile - part I. J Spinal Cord Med. 2014;37:693-702.

24. Eriks-Hoogland I, Hilfiker R, Baumberger M, Balk S, Stucki G, Perret C. Clinical assessment of obesity in persons with spinal cord injury: validity of waist circumference, body mass index, and anthropometric index. $\mathrm{J}$ Spinal Cord Med. 2011;34:416-22.

25. Cragg JJ, Ravensbergen HJ, Borisoff JF, Claydon VE. Optimal scaling of weight and waist circumference to height for adiposity and cardiovascular disease risk in individuals with spinal cord injury. Spinal Cord. 2015;53:64-8.

26. Maki KC, Briones ER, Langbein WE, Inman-Felton A, Nemchausky B, Welch M, et al. Associations between serum lipids and indicators of adiposity in men with spinal cord injury. Paraplegia. 1995;33:102-9.

27. Buchholz AC, Bugaresti JM. A review of body mass index and waist circumference as markers of obesity and coronary heart disease risk in persons with chronic spinal cord injury. Spinal Cord. 2005;43:513-8.

28. Gorgey AS, Dolbow DR, Gater DR. A model of prediction and cross-validation of fat-free mass in men with motor complete spinal cord injury. Arch Phys Med Rehabil. 2012;93:1240-5. 